\title{
Violencia intrafamiliar en pacientes pediátricos con problemas de salud mental
}

\author{
Cristina Haydée Arrom Suhurt ${ }^{\mathrm{I}}$, María del Pilar Fresco Arrom ${ }^{\mathrm{I}}$, Carmen Marina Arrom Suhurt ${ }^{\mathrm{II}}$, \\ Marcos Capurro', María Auxiliadora Arrom de Orrego ${ }^{\mathrm{II}}$, Andrés Claudelino Arce Ramírez ${ }^{\mathrm{I}}$, Margarita \\ Samudio ${ }^{\text {III }}$, Mirta Mercedes Romero Núñez ${ }^{\mathrm{I}}$ \\ I. Cátedra de Psiquiatría-Facultad de Ciencias Médicas de la Universidad Nacional de Asunción, Paraguay. \\ II. Centro para el Desarrollo de la Investigación Científica CEDIC/Díaz Gill, Paraguay. \\ III. Instituto de Investigaciones en Ciencias de la Salud-Universidad Nacional de Asunción (IICS-UNA), \\ Paraguay
}

Cómo referenciar este artículo/ How to reference this article:
Arrom C, Fresco MP, Arrom CM, Capurro M, Arrom MA, Arce A et al. Violencia intrafamiliar en pacientes pediátricos con problemas de salud mental. Mem. Inst. Investig. Cienc. Salud. 2015;13(3):816.

\section{RE S U M E N}

Existen distintas formas de violencia que se infligen contras niños y adolescentes en distintos ambientes, incluyendo los hogares, que producen consecuencias devastadoras en la salud mental y comunitaria. El objetivo del estudio fue analizar los diagnósticos de niños, niñas y adolescentes que consultaron al Servicio Ambulatorio de la Cátedra de Psiquiatría de la Facultad de Ciencias Médicas relacionados a diferentes tipos de violencia intrafamiliar. Es un estudio descriptivo, de corte transversal con muestreo no probabilístico. Se indagaron 145 fichas clínicas de pacientes infanto-juveniles que solicitaron atención psicológica a partir del 2010. Se analizaron los datos con SSPS 15.0 para Windows y el DSM-IV-TR para diagnósticos. Todos presentaron algún trastorno mental siendo los más frecuentes los trastornos del estado de ánimo 39\% (56), y entre ellos el más frecuente fue el depresivo mayor con $87,5 \%$. Otros fueron el trastorno por estrés post traumático en $11 \%$ (16) y trastornos de la conducta en $10,3 \%$ (15). El $71,4 \%$ (85) de la población estudiada recibió algún tipo de violencia. La forma de violencia más frecuente, sola o en combinación con otras, fue la psicológica con el 49,6\% (59) de los 85 que sufrieron maltrato. Se encontró una correlación estadísticamente significativa $(p=0,0058)$ entre el padecimiento de algún tipo de violencia y los trastornos del estado de ánimo. Este trabajo coincide con estimaciones nacionales e internacionales de niños que han sufrido algún tipo de violencia, así también los trastornos hallados son similares a los de otros trabajos.

Palabras clave: violencia doméstica, infanto juvenil, trastornos mentales.

\section{Domestic violence in pediatric patients with mental health problems}

\section{A B S T R A C T}

There are different types of violence that children and adolescents suffer in different environments, including homes, which produce devastating consequences. The objective was to analyze the diagnosis of children and adolescents who had consulted the Outpatient Service of the Department of Psychiatry of the Faculty of Medicine related to different types of domestic violence. It is a descriptive, cross-sectional study with non-probabilistic sampling. One hundred forty five medical records of children and adolescent who sought counseling from 2010 were used. Data were analyzed with SPSS 15.0 for Windows and DSM-IV-TR, for diagnosis. All cases presented some mental disorders. The most common disorders were mood disorders 39\% (56), and among them the major depressive disorder was the most frequent with $87.5 \%$. Other disorders found were post traumatic stress disorder in $11 \%$ (16) and behavioral disorders in $10.3 \%$ (15). From all the studied 
population, $71.4 \%$ (85) received some form of violence. The most frequent form, alone or in combination with another type, was the psychological with $49.6 \%$ (59) of the 85 who reported violence. A statistically significant correlation $(p=0.0058)$ was found between the condition of receiving violence and mood disorders. This work concurs with national and international data of children and adolescents who suffered violence, and the disorders found were similar to those of other works.

Keywords: domestic violence, child-youth, mental disorders

\section{INTRODUCCIÓN}

La violencia contra niños, niñas y adolescentes constituye una preocupación mundial. Su exposición como víctimas o testigos durante este periodo de la vida puede provocar trastornos sociales, emocionales, cognitivos y otras conductas que causan enfermedades, lesiones y problemas sociales (1). Los miembros de una familia, núcleo fundamental de las sociedades, no se encuentran excluidos de la posibilidad de padecerla o practicarla (2). Entendemos como violencia intrafamiliar todo acto u omisión realizado en el ambiente familiar por uno de sus miembros que produce daño físico, psicológico o sexual en otro de sus integrantes (3). Trasciende culturas y afecta a una importante población a nivel mundial. La violencia puede ser de tipo físico, psicológico, por negligencia o abandono, sexual o económica (1).

La alta incidencia de violencia intrafamiliar contra niños y adolescentes constituye uno de los principales problemas de salud pública de los países latinoamericanos, debido a las consecuencias negativas que produce en el proceso de desarrollo de la población que la experimenta $(4,5)$. Diferentes formas de violencia son practicadas por miembros de las familias, en tanto que los grupos más vulnerables son niños, mujeres, ancianos y discapacitados (2). Anualmente 80.000 niños y niñas mueren en América Latina a consecuencia de la violencia. El maltrato contra niños, en cualquiera de sus formas, no solo produce daño a la salud física y mental, sino que desequilibra los ambientes donde se desenvuelve su aprendizaje y produce pérdidas en lo referente a la igualdad entre géneros (6).

Sin saberlo, las familias con violencia familiar están produciendo un círculo vicioso, ya que al practicar castigos violentos como mecanismo de corrección, empujan a niños y niñas a continuar con comportamientos no deseados, lo cual aumenta sus probabilidades de volver a recibir castigos (7-9). En un estudio realizado por las universidades de Columbia y Princeton se encontró que aproximadamente el $28 \%$ de las madres reportó haber castigado físicamente a sus hijos durante el primer año de vida y esto aumentó hasta el $57 \%$ a los 3 años, $53 \%$ a los 5 años y $49 \%$ a los 9 años (10).

En Paraguay, la pobreza afecta a un $45 \%$ de los niños y $61 \%$ es víctima de algún tipo de violencia intrafamiliar, $35 \%$ de esos casos constituye violencia física grave. La familia juega un rol fundamental en la formación de niños, niñas y adolescentes y cuando se transforma en un lugar donde ocurre violencia y malos tratos, es importante ponerle fin a estos abusos (11). Un estudio de UNICEF reportó que $60 \%$ de los niños en edad escolar sufrió algún tipo de violencia en el hogar, $39 \%$ fue violencia física grave (golpes con objetos, patadas, quemaduras, y asfixia), 35\% violencia física leve (nalgadas con la mano y con objetos, cachetadas, tirones de pelo, pellizcos y la obligación de permanecer en posiciones incómodas) y el $13 \%$ padeció violencia psicológica (insultos, maldiciones, amenazas de abandono, entre otros) (11-13). En niños y niñas, la violencia física grave es la más frecuente, sin embargo las niñas reciben más maltrato psicológico que los varones (13). En un estudio probabilístico con estudiantes de 12 a 19 años del Departamento de Alto Paraná, se reportó que el $42.3 \%$ de los mismos había recibido cachetadas o puñetazos como forma de castigo de parte de sus padres, $39.3 \%$ golpes con objetos, $55.5 \%$ gritos y $30 \%$ insultos y groserías, y $8.6 \%$ reportó haber sufrido abuso sexual (14).

Diversas características se imprimen en la personalidad y la sintomatología que expresa la salud mental en víctimas de cualquier tipo de agresión. Es frecuente la dificultad para sentir placer, aplanamiento afectivo, alteraciones conductuales, problemas para establecer vínculos, baja autoestima, inseguridad, miedo, bajo rendimiento escolar por falta de concentración u otras alteraciones cognitivas $(15,16)$. Aquellos niños y adolescentes que crecen en ambientes violentos toman estas conductas como un modelo de comportamiento, lo cual sumado a la rabia y odio que experimentan, desembocan en trastornos conductuales centrados en agresividad y violencia (17). Así, un estudio que 
incluyó una población de más de 2.000 niños, niñas y adolescentes demostró que haber sido víctima de violencia en sus hogares fue un predictor de conductas violentas (18). Algunas de estas características, si se presentan juntas y persisten por un determinado tiempo llegan a configurar trastornos mentales graves como depresión, ansiedad, trastornos conductuales, psicosis y otros $(17,19)$. Un estudio realizado en la Cátedra de Psiquiatría de la Facultad de Ciencias Médicas de la Universidad Nacional de Asunción, con una población de 254 pacientes adultos que tenían antecedentes de violencia intrafamiliar, encontró que los mismos padecían principalmente trastornos del estado de ánimo, trastornos de ansiedad y de la personalidad (20).

Todos estos números, que constituyen una verdadera alarma sobre la situación de violencia por la que atraviesa el Paraguay y toda la región, y las graves consecuencias que puede acarrear el maltrato en el desarrollo y en el futuro de los niños y niñas resultan un importante justificativo, incluso imperativo, para profundizar el estudio de estos sucesos en nuestro país. La consulta ambulatoria constituye un momento oportuno para identificar, prestar ayuda y resolver situaciones de violencia familiar. En este trabajo se busca caracterizar la violencia intrafamiliar en una población infanto-juvenil que consultó al Servicio Ambulatorio de Psiquiatría de la Facultad de Ciencias Médicas.

\section{PACIENTES Y MÉTODO}

El estudio es descriptivo de corte transversal. Se estudiaron todas las fichas de pacientes en edad pediátrica (comprendida entre 3 y 18 años) que consultaron entre enero del 2010 y diciembre del 2012 en el Servicio Ambulatorio de la Cátedra de Psiquiatría. La información contenida en las fichas clínicas fue completada por el equipo de Psicólogas y Médicos Residentes del Servicio.

Para el objetivo del estudio que consistía en vincular la violencia intrafamiliar con padecimientos mentales de una población pediátrica del Servicio, fueron consignados datos sociodemográficos (edad, sexo, procedencia), impresiones diagnósticas en el Eje I y en el Eje II configuradas en base a criterios propuestos por el Manual Diagnóstico y Estadístico (DSM IV TR) de la Asociación Americana de Psiquiatría; Violencia Intrafamiliar utilizando la clasificación propuesta por el Grupo de trabajo sobre maltrato infantil dependiente del Ministerio de Salud de Chile (21) tipificada como: violencia o maltrato físico "toda lesión física no accidental producto de castigo único o repetido de magnitud y características variables"; de tipo emocional entendido como "hostigamiento verbal, habitual de un niño a través de insultos, criticas, descréditos y ridiculización, así como la indiferencia o rechazo implícito o explícito"; abandono o negligencia, considerado como "Falta de protección y cuidados físicos al niño, de parte de sus figuras de custodia, así como el abandono de sus necesidades evolutivas por falta de estimulación cognitivas que no se deben a carencias extremas de recursos socioeconómicos"; por último se define abuso sexual "entre un niño y un adulto, aguda o crónica, incluida la explotación sexual".

Un total de 145 pacientes consultó al Servicio en el período de referencia manifestando diferentes sintomatologías. De este grupo, $82 \%$ (119) tenía información referente a exposición a alguna forma de violencia o maltrato en el ámbito familiar; 71,4 (85) de los cuales fue víctima en algunas de las formas de violencia y 28,6 (34) no; 92,4\% (134) padecía algún trastorno mental. Se procedió a analizar los distintos tipos de violencia padecida por el grupo de 119 pacientes con los diferentes trastornos que presentaron al llegar al Servicio.

Las variables fueron cargadas en una base de datos en Excel y para el análisis de la información se utilizó el paquete estadístico SPSS versión 15.0 para Windows 7. Los resultados se expresan en forma de frecuencia absoluta y relativa; para indagar posibles asociaciones entre tipo de violencia y trastornos se utilizó la prueba de chi cuadrado a un nivel de significancia de 0,05.

\section{RESULTADOS}

Del total del grupo (145) 59\% (86) de los pacientes tenía entre 12 y 18 años, 30\% (44) entre 6 y 11 , y $10 \%$ (15) menos de 5 . Hubo leve predominio del sexo femenino $(51,0 \%)$. El 57\% (82) procedía de Asunción (Tabla 1). 
Tabla 1. Características socio-demográficas de los pacientes pediátricos del Servicio Ambulatorio de la Cátedra de Psiquiatría de la Facultad de Ciencias Médicas. n=145

\begin{tabular}{lll}
\hline Características socio-demográficas & $\mathbf{n}$ & $\%$ \\
\hline Edad (años) & & \\
3 a 5 & 15 & 10,4 \\
6 a 8 & 19 & 13,1 \\
9 a 11 & 25 & 17,2 \\
12 a 14 & 38 & 26,2 \\
15 a 18 & 48 & 33,1 \\
Sexo & & \\
$\quad$ Masculino & 71 & 49 \\
$\quad$ Femenino & 74 & 51 \\
Procedencia & & \\
$\quad$ Asunción & 82 & 56,55 \\
$\quad$ Central & 39 & 26,90 \\
Interior & 23 & 15,86 \\
$\quad$ Exterior & 1 & 0,69 \\
\hline
\end{tabular}

El 92,4\% (134) de los individuos estudiados presentó algún trastorno mental, de los cuales en el 39\% (56) se observó algún trastorno del estado de ánimo, seguido de trastorno por estrés post traumático en el $11 \%(16)$ y trastornos de la conducta en el $10,3 \%(15)$, entre los más frecuentes. El 7,5\% (11) de pacientes tuvo diagnóstico aplazado por no presentar trastorno al momento de la consulta. Dos pacientes tenían trastornos psicóticos, lo que representa el $1,4 \%$, de los cuales 1 fue diagnosticado con esquizofrenia paranoide (Tabla 2).

Tabla 2. Tipo de trastornos mentales en pacientes pediátricos del Servicio Ambulatorio de la Cátedra de Psiquiatría de la Facultad de Ciencias Médicas. $\mathrm{n}=145$

\begin{tabular}{lll}
\hline Tipo de trastornos mentales & $\mathbf{n}$ & $\mathbf{\%}$ \\
\hline Trastornos del estado de ánimo con y sin sintomatología ansiosa & 56 & 39 \\
Trastornos por estrés post traumático & 16 & 11 \\
Trastorno p/déficit de atención y comportamiento perturbador & 15 & 10,3 \\
Problemas de relación & 7 & 4,8 \\
Trastornos relacionados con substancias & 6 & 4,1 \\
Trastornos adaptativos & 4 & 2,7 \\
Trastornos del aprendizaje no especificado & 4 & 2,7 \\
Trastorno generalizado del desarrollo & 3 & 2,1 \\
Otros trastornos de la infancia, niñez y adolescencia no especificados & 3 & 2,1 \\
Trastornos psicóticos/no especificados & 2 & 1,3 \\
Diagnóstico aplazado al momento de la consulta & 11 & 7,5 \\
\hline
\end{tabular}

En los 56 individuos con trastornos del estado del ánimo, el trastorno depresivo mayor (leve, moderado o grave) fue el más frecuente con $87,5 \%$. Entre los 15 pacientes con trastornos conductuales se encontró como más frecuentes al trastorno por déficit de atención e hiperactividad y el trastorno negativista desafiante con un $26 \%$ cada uno (Tabla $3)$. 
Tabla 3. Trastornos mentales en pacientes pediátricos del Servicio Ambulatorio de la Cátedra de Psiquiatría de la Facultad de Ciencias Médicas.

\begin{tabular}{ll}
\hline Trastornos & $\mathbf{n}(\mathbf{\%})$ \\
\hline Trastornos del estado de ánimo & \\
Trastorno depresivo mayor leve & $33(58,9)$ \\
Trastorno depresivo mayor moderado & $8(14,3)$ \\
Trastorno depresivo mayor grave & $6(10,7)$ \\
Trastorno depresivo mayor +trastorno límite de la personalidad & $1(1,8)$ \\
Trastorno depresivo mayor/leve + trastorno relacionado con otra sustancia $(0$ & $1(1,8)$ \\
desconocida) no especificado & $1(1,8)$ \\
Trastorno depresivo no especificado & $2(3,6)$ \\
Trastorno distímico de inicio temprano & $4(7,1)$ \\
Duelo &
\end{tabular}

\section{Trastornos de la conducta}

Déficit de atención con hiperactividad, tipo combinado

Negativista desafiante

Disocial

Comportamiento perturbador no especificado

Déficit de atención y comportamiento perturbador/ tipo con predominio de déficit de atención

\section{Otros trastornos psicóticos/ no especificados}

Comportamiento antisocial en la niñez y la adolescencia

Factores psicológicos que afectan al estado físico/rasgos de personalidad o estilo de afrontamiento que afectan a una enfermedad médica

Trastorno del estado de ánimo debido a enfermedad médica (epilepsia)

Trastorno de ansiedad generalizada

Anorexia nerviosa

Exhibicionismo

Retardo mental /moderado

Retraso mental de gravedad no especificada

Trastorno del lenguaje expresivo

Ansiedad por separación

Mutismo selectivo

Se tuvo información sobre violencia en 119 pacientes, de los cuales el 71,4\% $(n=85)$ refirió algún tipo de violencia. La forma de violencia más frecuente fue la psicológica en el $49,6 \%(n=59)$, sola o en combinación con otras. La violencia física sola o en combinación con otros tipos se presentó en el $47 \%$ de los casos (Tabla 4 ). 
Tabla 4. Tipo de violencia en pacientes pediátricos del Servicio Ambulatorio de la Cátedra de Psiquiatría de la Facultad de Ciencias Médicas. $n=119$

\begin{tabular}{|c|c|c|c|}
\hline Tipo de violencia & & $\mathbf{n}$ & $\%$ \\
\hline \multirow{6}{*}{$\begin{array}{l}\text { Psicológica } \\
n=59(49,6 \%)\end{array}$} & Psicológica y física & 32 & 26,9 \\
\hline & Psicológica & 19 & 16,0 \\
\hline & Psicológica, física, y abuso sexual & 3 & 2,5 \\
\hline & Psicológica y por negligencia/abandono & 3 & 2,5 \\
\hline & Psicológica, física, y por negligencia/abandono & 1 & 0,8 \\
\hline & Psicológica y abuso sexual & 1 & 0,8 \\
\hline \multirow{3}{*}{$\begin{array}{l}\text { Física } \\
n=19(16 \%)\end{array}$} & Física & 14 & 11,8 \\
\hline & Física y abuso sexual & 4 & 3,4 \\
\hline & Física y negligencia/abandono & 1 & 0,8 \\
\hline $\begin{array}{l}\text { Abuso sexual } \\
n=6(5 \%)\end{array}$ & Abuso sexual & 6 & 5,0 \\
\hline $\begin{array}{l}\text { Negligencia/abandono } \\
n=1(0,8 \%)\end{array}$ & Por negligencia o abandono & 1 & 0,8 \\
\hline Sin violencia & & 34 & 28,6 \\
\hline
\end{tabular}

De los 85 pacientes que reportaron haber sufrido algún tipo de maltrato, el $53 \%(n=45)$ presentaba trastorno del estado del ánimo, siendo la asociación estadísticamente significativa (valor $\mathrm{p}=0,0058$ ) (Tabla 5). En la Tabla 6 se muestran los tipos de violencia y los trastornos mentales de los pacientes.

Tabla 5. Asociación entre tipo de violencia y trastornos presentados en pacientes pediátricos del Servicio Ambulatorio de la Cátedra de Psiquiatría de la Facultad de Ciencias Médicas. n=119

\begin{tabular}{llll}
\hline Trastornos & \multicolumn{3}{c}{ Violencia } \\
& Sufrió & No sufrió & Total \\
\hline del estado de ánimo /combinado & $\mathbf{4 5}(\mathbf{5 3})$ & $7(20,6)$ & 52 \\
por estrés post traumático & $13(15,3)$ & $2(6)$ & 15 \\
por déficit de atención y comportamiento perturbador & $8(9,4)$ & $4(11,7)$ & 12 \\
Otros & $19(22,3)$ & $\mathbf{2 1 ( 6 1 , 8 )}$ & 40 \\
Total & 85 & 34 & 119 \\
\hline
\end{tabular}


Tabla 6. Asociación entre tipo de violencia y trastornos presentados en pacientes pediátricos del Servicio Ambulatorio de la Cátedra de Psiquiatría de la Facultad de Ciencias Médicas. n=119

\begin{tabular}{|c|c|c|c|c|c|}
\hline Con violencia & $\begin{array}{l}\text { del estado } \\
\text { de ánimo/ } \\
\text { combinado }\end{array}$ & $\begin{array}{l}\text { por estrés } \\
\text { post } \\
\text { traumático }\end{array}$ & $\begin{array}{l}\text { Trastornos } \\
\text { por déficit de } \\
\text { atención y } \\
\text { comportamiento } \\
\text { perturbador }\end{array}$ & Otros & Total \\
\hline Psicológica y física & $17(53,1)$ & $5(15,63)$ & $2(6,25)$ & $8(25)$ & 32 \\
\hline Psicológica & $11(52,4)$ & $1(4,8)$ & $1(4,8)$ & $8(38,1)$ & 21 \\
\hline $\begin{array}{l}\text { Psicológica y } \\
\text { negligencia/abandono }\end{array}$ & - & $1(33,3)$ & - & $2(66,7)$ & 3 \\
\hline $\begin{array}{l}\text { Psicológica, física y abuso } \\
\text { sexual }\end{array}$ & $2(66,7)$ & - & - & $1(33,3)$ & 3 \\
\hline Psicológica y abuso sexual & $1(50)$ & $1(50)$ & - & - . & 1 \\
\hline $\begin{array}{l}\text { Psicológica, física, y } \\
\text { negligencia/abandono }\end{array}$ & - & . & $1(100)$ & & 1 \\
\hline Física & $10(62,5)$ & $1(6,25)$ & $3(18,75)$ & $2(3,4)$ & 16 \\
\hline Abuso sexual & $3(60)$ & $2(40)$ & - & - & 5 \\
\hline Física y abuso sexual & $1(33,3)$ & $2(66,7)$ & - & . & 3 \\
\hline Negligencia/abandono & - & . & $1(100)$ & - & 1 \\
\hline $\begin{array}{l}\text { Física y negligencia/ } \\
\text { abandono }\end{array}$ & - & . & - & $1(100)$ & 1 \\
\hline
\end{tabular}

\section{DISCUSIÓN}

En concordancia con la información obtenida acerca de la frecuencia de niños y niñas del Paraguay que han recibido algún tipo de maltrato (13), este trabajo revela que una semejante proporción de las niñas, niños y adolescentes que forman parte del grupo de estudio han sido víctimas de violencia. Este dato no solo coincide con estadísticas nacionales, sino también con estudios de referencia de la región. En la mayoría de los países de América del Sur se observan cifras similares (22). En Argentina, un estudio de 450 estudiantes universitarios reveló que la mitad había sido víctima de castigos físicos en su infancia (23); en Chile, de 1.525 niños y adolescentes tres cuartos recibió violencia por parte de sus padres (24). Lo mismo en Uruguay, un estudio de 1.100 familias reveló que la mayoría de los adultos entrevistados aplicaba alguna forma de violencia psicológica o física hacia un niño en su hogar (25).

En cuanto a los tipos de violencia intrafamiliar, la más frecuentemente practicada entre la población víctima fue la combinación de maltrato físico y psicológico, seguido de violencia psicológica. No obstante, al considerar las formas de violencia solas o combinadas, la psicológica fue la más frecuente entre los niños y adolescentes que sufrieron una o más formas de violencia; este dato diverge de lo propuesto por otras investigaciones de la región que estiman que la violencia física, sola o combinada, es la más practicada $(17,26,27)$. Esta diferencia podría deberse a la amplia y desafortunada aceptación social que tienen los castigos físicos como herramientas correctivas en la educación de los niños en los hogares paraguayos $(12,13)$, lo que a su vez podría hacer que algunos pacientes no se manifiesten como víctimas de este tipo de violencia por encontrarse validados culturalmente como mecanismos educativos.

Los trastornos del estado de ánimo con y sin características ansiosas, fueron los diagnósticos más frecuentes, representados en su mayoría por el trastorno depresivo 
mayor. Al igual que lo propone la literatura sobre los trastornos mentales más frecuentes en niños víctimas de violencia intrafamiliar (18), tras la depresión se ha hallado el trastorno por estrés post traumático. Entre los trastornos externalizados, clasificación utilizada para aquellos trastornos dominados por síntomas de conducta disruptiva (18), los más frecuentes fueron el trastorno por déficit atencional e hiperactividad y los trastornos disocial y negativista desafiante. Merece la pena mencionar que todo esto no se ha distanciado de lo que la literatura nacional e internacional de salud mental infanto-juvenil propone como las psicopatologías más frecuentemente relacionados al padecimiento de violencia, tanto de aquellos trastornos externalizados (caracterizados por ira y conductas disruptivas) como los internalizados (dominados por síntomas de ansiedad, depresión o disociación) $(18,20,27,28)$. Al asociar los trastornos mentales hallados con los diversos tipos de violencia se encontró una correlación estadísticamente significativa ( $p$ menor a 0,0058 ) entre el padecimiento de algún tipo de violencia y trastornos del estado de ánimo, en consonancia con otros datos de nuestro país (27).

Parte de esta sintomatología, sobre todo de características externalizadas, podrían explicarse por la característica propia del proceso adolescente normal, que requiere ejercicio de la rebeldía; ésta, contextualizada en una larga historia de autoritarismo y baja tolerancia a expresiones de libertad, resultaría en la utilización de más violencia hacia esta población. Por lo tanto, para poder profundizar en la compresión del desarrollo de trastornos mentales como consecuencia de la violencia es necesario ampliar el tamaño de la muestra e incorporar el estudio de otros factores como predisponentes o desencadenantes, lo que constituye una limitación del presente estudio.

Merece la pena destacar el impacto de este estudio al constatar que casi dos tercios de las familias de los pacientes incluidos en la población estudiada, han utilizado alguna o varias modalidades de violencia como forma de castigo, dato que no resulta difícil de contextualizar en el Paraguay teniendo en cuenta de que tales prácticas gozan de un apoyo social y se justifica en distintos ámbitos como metodología de corrección (13) del comportamiento disruptivo o inadecuado de los niños, niñas y adolescentes paraguayos.

\section{REFERENCIAS BIBLIOGRAFICAS}

1. Ministerio de Desarrollo Social. Abordajes frente a la violencia familiar desde una perspectiva de género y de infancia [Internet]. Buenos Aires: Ministerio de Desarrollo Social de la Provincia de Buenos Aires; 2009. Serie: Fortalecimiento de Equipos de Trabajo en Género [citado 10 Jun 2013]. Disponible en PDF: http://www.unicef.org/argentina/spanish/ abordajes.pdf

2. Almenares Aleaga M, Louro Bernal I, Ortiz Gómez MT. Comportamiento de la violencia intrafamiliar. Rev Cubana Med Gen Integr. 1999; 15(3):285-92.

3. Rodríguez Sánchez I, Torres Lugo DJ, Castillo Ledo I. La violencia intrafamiliar y su repercusión en la salud mental del niño y del adolescente. Rev.psiquiatr.psicol niño adolesc. 2007; 7(1):20-37.

4. Kliksberg B. Evolución, de la relación del niño, la niña y el adolescente con su familia. 2004. /Internet/. En: Instituto Interamericano del Niño. XIX Congreso Panamericano del Niño. México: OEA. [citado 6 Jun 2013]. Disponible en: www.scm.oas.org/idms_public/SPANISH/h ist_10/DIL00222S02.DOC

5. Barcelata Eguiarte BE, Alvarez Antillón EI. Patrones de interacción familiar de madres y padres generadores de violencia y maltrato infantil. Act.Colom.Psicol. 2005; $8(1): 285-92$.

6. Naciones Unidas, Asamblea General. Promoción y protección de los derechos de los niños. Informe del experto independiente para el estudio de la violencia contra los niños, de las Naciones Unidas [Internet]. Naciones Unidas; 2006 agosto. A/61/299. [citado 6 Jun 2014]. Disponible en: http://www.unicef.org/violencestudy/repo rts/SG_violencestudy_sp.pdf

7. Gershoff ET. More harm than good: a summary of scientific research on the intended and unintended effects of corporal punishment on children. Law \& Contemp Probs. 2010; 73(2):31-56.

8. Berlin LJ, Ispa JM, Fine MA, Malone PS, Brooks-Gunn J, Brady-Smith $C$, et al. Correlates and consequences of spanking and verbal punishment for low-income white, african american, and mexican american toddlers. Child Dev. 2009; 80(5):1403-20.

9. Regalado $M$, Sareen $H$, Inkelas $M$, Wissow LS, Halfon N. Parents' discipline of young children: results from the National Survey of Early Childhood Health. Pediatrics. 2004. 113(6 Suppl): 1952-8. 
10. Michael MacKenzie. Castigar con azotes desencadena un círculo vicioso: MedlinePlus [Internet]. Nueva York-USA: Michael MacKenzie-Facultad de Trabajo Social de la Universidad de Columbia; [citado 12 marzo 2014]. Disponible en: http://www.efekeze.com/index.php/es/mat erno-infantil/item/739-castigar-con-azotesdesencadena-un-circulo-viciosomedlineplus

11. UNICEF. Pobreza afecta al $45 \%$ de los niños paraguayos y la violencia al $61 \%$. Paraguay.com. /Internet/. Asunción: 2012 /citado 15 mayo 2015/. Disponible en: http://www.paraguay.com/nacionales/pobr eza-afecta-al-45-de-los-ninos-paraguayosy-la-violencia-al-61-82053

12. Naciones Unidas [Internet]. AsunciónParaguay: Movimiento Mundial por la Infancia y Secretaría Nacional de la Niñez y la Adolescencia; [citado 4 Jun 2013]. Disponible en: http://www.unicef.org/paraguay/spanish/ py_hoja_datos_violencia_abr11.pdf

13. Gaona $\bar{O}$. Estudio sobre maltrato infantil en el ámbito familiar Paraguay [Internet]. Asunción: BECA, UNICEF; 2011 /citado 11 Jun 2013/. Disponible: http://www.unicef.org/paraguay/spanish/ py_resources_Estudio_Maltrato.pdf

14. Ruoti M, Arrom C. Diferentes formas de violencia padecidas por adolescentes durante su crianza. Revista Paraguaya de Psiquiatría 2015; 3(1):50-6.

15. Trujano $\mathrm{P}$, Martínez AE, Camacho, SI. Varones víctimas de violencia doméstica: un estudio exploratorio acerca de su percepción y aceptación. Diversitas /online/. 2010;6(2):339-54. Available from:

http://www.scielo.org.co/scielo.php?scrip $\mathrm{t}=\mathrm{sci}$ arttext\&pid=S1794

99982010000200010\&lng=en\&nrm=iso. ISSN 1794-9998.

16. Kliksberg B. La familia en América Latina: Realidades, interrogantes y perspectivas. ; 2004. /Internet/. México: OEA, Instituto Interamericano del Niño En: XIX Congreso Panamericano del Niño. [citado 8 Agosto 2013]. Disponible en PDF en: http://www.iin.oea.org/iin/cad/actualizaci on/pdf/1_2/basica/La\%20familia\%20en\% 20Am\%C3\%A9rica\%20Latina-

Realidades,\%20interrogantes\%20y\%20pe rsp.,Kliksberg,\%20XIX\%20Congreso\%20P anamericano\%20del\%20Ni\%C3\%B10,\%2 Oesp.pdf

17. Pérez C. La violencia intrafamiliar y su incidencia en el desarrollo de niños y niñas. 2004. /Internet/. Conferencia Magistral. En: Instituto Interamericano del Niño. XIX Congreso Panamericano del Niño. México: OEA, Instituto Interamericano del Niño; [citado 8 Agosto 2013]. Disponible en PDF en: www.scm.oas.org/idms_public/SPANISH/h ist_10/DIL00222S02.DOC
18. Viola L. Repercusión de la violencia doméstica en la salud mental infantil: valoración del daño psíquico. Rev Psiquiatr Urug. 2010. 74(1):73-83.

19. Spilsbury JC, Kahana S, Drotar D, Creeden R, Flannery DJ, Friedman S. Profiles of behavioral problems in children who witness domestic violence. Violence Vict. 2008; 23(1):3-17.

20. Fresco MP, Arrom C, Samudio M, Arrom CM, Capurro M, Arrom MA, et al. Psicopatología en adultos con antecedentes de violencia intrafamiliar. Revista Paraguaya de Psiquiatría. 2015;3(1):8-12.

21. Vizcarra MB, Cortés JM, Bustos LM, Alarcón ME, Muñoz SN. Maltrato infantil en la ciudad de Temuco: estudio de prevalencia y factores asociados. Rev Méd de Chile. $2001 ; 129(12): 1425-32$

22. Pinheiro PS. Informe mundial sobre la violencia contra los niños y niñas /Internet/. Ginebra: Fondo de las Naciones Unidas para la Infancia; 2005. [citado 11 Jun 2013]. Disponible en PDF en:

http://www.unicef.org/lac/Informe_Mundi al_Sobre_Violencia_1\%281\%29.pdf

23. Giberti E. Abuso sexual y malos tratos contra niños, niñas y adolescentes: perspectiva psicológica y social. Buenos Aires: Espacio Editorial; 2005.

24. Larraín S, Bascuñan C. Maltratao infantil y relaciones familiares en Chile: análisis comparativo 1994-2000-2006 /Internet/. Santiago de Chile: Fondo de las Naciones Unidas para la Infancia; 2008. [citado 13 Jun 2013]. Disponible en PDF en: http://www.unicef.cl/web/wp-

content/uploads/doc_wp/maltrato_parawe b.pdf

25. De los Campos H, Solari M, González M. Prácticas de crianza y resolución de conflictos familiares: prevalencia del maltrato intrafamiliar contra niñas, niños y adolescentes /Internet/. Montevideo: Ministerio de Desarrollo Social, Programa Infancia, Adolescencia y Familia; 2008. [citado 8 Jul 2013]. Disponible en PDF en:

http://resourcecentre.savethechildren.se/s ites/default/files/documents/3653.pdf

26. Larraín S., Bascuñan C. Maltrato infantil: una dolorosa realidad puertas adentro. Desafíos 2009;9: 4-9

27. Arrom C, Fresco MP, Arrom CM, Arce A, Arrom MA, Samudio $M$, et al. Violencia intrafamiliar. Revista Paraguaya de Psiquiatría. 2015; 3(1):38-41.

28. Sadock BJ, Sacock VA, Ruiz P. Kaplan and sadock's synopsis of psychiatry: behavioral sciences/clinical psychiatry. $11^{\text {th }}$ ed. Nueva York: Wolters Kluwer; 2014. 